\title{
Equilíbrio e Coordenaçáo Motora em Escolares Praticantes e Náo Praticantes de Atividades Física e/ou Lúdica Extra-Escolar
}

\author{
Balance and Motor Coordination In Students Who Practice Physical andlor Playful or After-School \\ Cristina dos Santos Cardoso de Sá1, Bruna Carvalho ${ }^{2}$, Carla Mazzitelli ${ }^{3}$
}

\section{RESUMO}

Introduçáo. Fatores externos influenciam o processo de aquisiçôes de habilidades motoras em cada idade. Objetivo. Este estudo verificou a influência da atividade física e/ou lúdica direcionada sobre o desenvolvimento motor (DM) de escolares, e se os hábitos de vida diária destes influenciam a aquisição das habilidades motoras. Método. Noventa escolares, entre 8 e 12 anos de idade, foram divididas em grupos A e B, praticantes e não praticantes de atividade física e/ou lúdica direcionada, respectivamente. A coordenaçáo motora foi avaliada pelo teste de KTK e o equilíbrio pela escala de Equilíbrio Pediátrica. Para verificar o nível de atividade física, os pais responderam o questionário PAQ-C, e o questionário de Classificação Econômica Brasil (CCEB) verificou o nível sócio econômico. Resultados. Os resultados indicaram que o grupo A obteve melhor desempenho em relação à coordenação e equilíbrio, são escolares ativos, e permanecem menos tempo assistindo TV. Conclusáo. Os hábitos de vida de crianças de 8 a 12 anos, principalmente no que se refere a um estilo de vida ativo, observado pela prática de atividades física direcionada e/ou lúdica pode influenciar o desenvolvimento de habilidades motoras em crianças desta idade.

Unitermos. Desempenho Psicomotor, Equilíbrio, Coordenação, Criança.

Citaçáo. Sá CSC, Carvalho B, Mazzitelli C. Equilíbrio e Coordenação Motora em Escolares Praticantes e Não Praticantes de Atividades Física e/ou Lúdica Extra-Escolar.

\begin{abstract}
Introduction. External factors influencing the process of acquisition of motor abilities at each age. Objective. This research has verified the influence of physical and/or directed play activities directed on the motor development (MD) of students, and whether daily-life habits have any influence on the acquisition of motor skills. Method. Ninety students, between 8 and 12 years old, were divided in two groups: A and $\mathrm{B}$, practitioners and non-practitioners of physical and/or directed play activities, respectively. The motor coordination was measured by the KTK test and balance by the scale of the Pediatric Balance. In order to check the level of physical activity, parents completed the PAQ-C questionnaire and the questionnaire of the Brazilian Economic Classification Scheme (CCEB) classified the socioeconomic level. Results. The results indicated that group A had the best performance in relation to coordination and balance, students are active, and spend less time watching TV. Conclusion. That life habits of children aged between 8-12 years, principally in regard to active lifestyle, observed by the practice of physical and/or directed play activities can influence the development of motor skills in children of this age.
\end{abstract}

Keywords. Psychomotor Performance, Balance, Coordination, Child.

Citation. Sá CSC, Carvalho B, Mazzitelli C. Balance and Motor Coordination In Students Who Practice Physical and/or Playful or After-School.

\footnotetext{
Trabalho realizado na Universidade Metodista de São Paulo em parceria com a Universidade Federal de Sáo Paulo.

1.Fisioterapeuta, Doutora Professora do Departamento de Ciências do Movimento Humano - Universidade Federal de São Paulo - UNIFESP, Campus Baixada Santista, Santos-SP, Brasil.

2.Fisioterapeuta, especialista em Fisioterapia Neurológica pela Universidade Metodista de São Paulo, São Paulo-SP,Brasil

3.Fisioterapeuta, Doutora, Professora do Curso de Fisioterapia Universidade

Metodista de São Paulo, São Paulo-SP, Brasil.
}

Endereço para correspondência: Cristina dos Santos Cardoso de Sá Av. Dona Ana Costa 95, Vila Mathias CEP 11060-001, Santos-SP, Brasil. E-mail: cristina.sa@unifesp.br

Original

Recebido em: 15/07/13

Aceito em: 12/03/14

Conflito de interesses: não 


\section{INTRODUÇÃO}

Desenvolvimento Motor é um processo sequencial relacionado à idade cronológica, que retrata a interação entre as características do indivíduo, da tarefa e do ambiente, e é influenciado por fatores socioculturais, intelectuais e emocionais ${ }^{1,2}$. Durante a infância, especialmente, no início da idade escolar, há um importante incremento das habilidades motoras, que permite a criança o domínio do seu corpo em diversas atividades ${ }^{3-6}$.

Para a criança atingir o seu completo desenvolvimento motor, é necessário que vivencie diversas atividades motoras. A experiência, juntamente com a maturação e crescimento, irá propiciar significativamente o desenvolvimento motor de várias capacidades físicas e melhoria no desempenho motor. $\mathrm{O}$ cotidiano da criança influencia diretamente a aquisição e refinamento das habilidades motoras. Rosa Neto et $\mathrm{al}^{6}{ }^{6}$, acredita que, o fato de a criança explorar o ambiente por meio de atividades motoras (como o exercício físico e o jogo) ou pelo desempenho de habilidades motoras implicará em modificações no seu desenvolvimento físico, perceptivo-motor, moral e afetivo.

Estudo realizado com crianças de cinco a sete anos de uma organização não governamental, que praticam rotineiramente no contra período escolar, dança, música, brincadeiras recreativas e lúdicas e atividade física direcionada, revelou melhor desempenho motor nas atividades envolvendo equilíbrio e coordenação motora ${ }^{7}$. Portanto o preenchimento adequado do tempo livre com atividades lúdica e/ou física direcionada é uma importante ferramenta para o desenvolvimento, aprendizagem e refinamento das habilidades motoras 5 .

Nos últimos anos, mudanças sociais, econômicas e espaciais têm interferido no comportamento das crianças. A falta de experiência motora e da prática de programas de atividades físicas direcionadas são grandes causadores do sedentarismo em crianças, adolescentes e adultos, que compromete o desempenho motor, em atividades que envolvem principalmente a coordenação motora e o equilíbrio, levando no caso de crianças e adolescentes, a prejuízos do desenvolvimento das habilidades motoras e da saúde, principalmente no que se refere a doenças metabólicas ${ }^{8-12}$. A prática de atividade motora tem efeito considerável sobre o desenvolvimento físico; habilidades locomotoras, manipulativas e perceptivo-motoras, e fornece experiência que estimula o amadurecimento dos aspectos sócio afetivo. Crianças que apresentam baixos níveis de atividade motora prejudicam o refinamento de suas habilidades motoras, perceptivo-motoras e podem comprometer a aprendizagem escolar ${ }^{6,13,14}$.

Entende-se que a promoção da saúde na infância, desenvolvimento de hábitos saudáveis e aumento do repertório motor podem ser obtidos por meio da atividade física e/ou lúdica que incluem elementos básicos da motricidade grosseira e fina, equilíbrio estático e dinâmico, esquema corporal, organização espacial, temporal e lateralidade. Portanto, há uma preocupação com o atual estilo de vida das pessoas e as consequências que a falta de oportunidades de exploração dos movimentos podem causar, como: descoordenação da movimentação, problemas na aprendizagem escolar e no desenvolvimento sócio afetivo e outros.

O objetivo deste estudo foi avaliar a influência das atividades motoras (atividades físicas e/ou lúdicas) e dos hábitos de vida sobre o equilíbrio e coordenação motora de escolares de oito a 12 anos.

\section{MÉTODO}

\section{Participantes e Local}

Noventa escolares entre oito e 12 anos de idade regularmente matriculadas em escolas da rede pública de São Caetano do Sul e de São Paulo. Foram divididas em dois grupos, praticantes de atividade lúdica e/ou física direcionada (grupo A) e não praticantes de atividade lúdica e/ou física direcionada (grupo B). Cada grupo foi formado por 10 escolares de oito anos, 10 escolares de nove anos, 11 escolares de 10 anos, 10 escolares de 11 anos e quatro escolares de 12 anos. Foram excluídos escolares com patologias ortopédicas e neurológicas, ou que apresentassem deficiência visual ou auditiva.

Os escolares do grupo A realizaram as atividades físicas e/ou lúdicas propostas regularmente na escola, e no contra período escolar, permaneciam em uma instituição não governamental por quatro horas diárias, na qual realizavam atividades físicas e/ou lúdicas (aulas de dança, música, circo, atividades recreacionais e lúdicas). Os escolares do grupo B apenas realizaram as atividades físicas e/ou lúdicas propostas pela escola regular no período em que o escolar frequentava a escola. 
Este estudo seguiu os princípios éticos de pesquisa com seres humanos contidos na Declaração de Helsinki (1964, reformulada em 1975, 1983, 1989, 1996, 2000 e 2008), da World Medical Association, e foi aprovado pelo Comitê de Ética em Pesquisa: CEP-UMESP, prot. $n^{\circ}$. 290299-09 CAEE: 0067.0.214.000-09.

\section{Materiais}

\section{Estadiômetro e balança digital foram utilizados na re- alização das medidas antropométricas}

Utilizou-se a Escala de Equilíbrio Pediátrica - Versão Modificada da Escala de Berg ${ }^{15}$, e o teste de Coordenação Corporal para Crianças - $\mathrm{KTK}^{16}$ para avaliar o equilíbrio e a coordenação das crianças. A classificação dos autores alemães para os escolares apresenta limitaçóes para a população brasileira. Desse modo a classificação foi realizada adaptação das atribuiçôes da classificação da coordenação para a população brasileira ${ }^{17}$.

Para realização dos testes de equilíbrio utilizou-se os seguintes materiais: fita métrica, fita adesiva, cronômetro, cadeira sem apoio e banco de madeira (escala de equilíbrio pediátrica - Berg modificada ${ }^{16}$; três traves de madeira de 3 metros de comprimento e $3 \mathrm{~cm}$ de altura, com larguras de $6 \mathrm{~cm}, 4,5 \mathrm{~cm}$ e $3 \mathrm{~cm}$, duas plataformas de $25 \times 25 \times 5 \mathrm{~cm}, 12$ blocos de espumas, medindo cada um $50 \times 20 \times 5 \mathrm{~cm}$ e um sarrafo divisório de $60 \times 4 \times 2 \mathrm{~cm}$ e um step de $10 \mathrm{~cm}$ de altura $^{17}$.

O questionário PAQ-C traduzido por Silva e Malina ${ }^{18}$ para avaliar o nível de atividade física regular das crianças e o Questionário Classificação Econômica Brasil (CCEB) que consiste de duas etapas, a primeira relacionada ao grau de instrução dos familiares do escolar (nível de escolaridade), e a segunda refere-se aos itens de posse e quantidade de cada um.

\section{Procedimentos}

Os pais e/ou responsáveis pelos escolares assinaram o termo de consentimento livre esclarecido para autorizarem a participação do seu filho ou filha no estudo, e responderam o Questionário de classificação econômica Brasil.

Posteriormente, os pais responderam o questionário PAQ-C constituído de 13 questóes, nove questôes sobre prática de esportes e jogos, atividades físicas na escola ou de lazer em finais de semana. Cada questão tem valor de 1 a 5, o escore final é obtido pela média das questóes, assim podemos classificá-las como (1) muito sedentária; (2) e (3) indicam as categorias sedentárias, (4) ativas e (5) muito ativas. $\mathrm{O}$ restante das questóes inclui perguntas sobre o nível comparado de atividades, sobre a média diária do tempo que assistem TV e sobre a presença de alguma doença que impeça a atividade física regular na semana avaliada. Os pais foram instruídos pelo examinador antes de iniciar o preenchimento do PAQ-C.

Os escolares com idade de oito a 12 anos realizaram primeiramente avaliação antropométrica, e na sequência o teste da escala de equilíbrio pediátrica- versão modificada da escala de Berg, que consiste em 14 itens para avaliar o equilíbrio estático e dinâmico.

Posteriormente a avaliação do equilíbrio, os escolares realizaram o teste de Coordenação Corporal para Crianças - $\mathrm{KTK}^{7,16}$, que consiste na execução de quatro tarefas para avaliar o equilíbrio e coordenação.

A primeira tarefa - Trave de Equilíbrio - avalia a estabilidade do equilíbrio em marcha para trás sobre a trave. São utilizadas três traves, na qual cada escolar anda de costas sobre cada uma delas, e é contado o número de passos realizados nas traves.

A segunda tarefa - Salto Monopodal - avalia a coordenação dos membros inferiores, energia dinâmica e força. O escolar é instruído a saltar blocos de espuma, salto monopodal, com um membro inferior de cada vez, esses saltos são pontuados com base na tentativa. A quantidade de blocos é determinada pela idade, escolares de oito anos começam com três blocos (altura de $15 \mathrm{~cm}$ ), de nove a dez anos com cinco blocos (altura de $25 \mathrm{~cm}$ ) e de 11 a 12 anos com sete blocos (altura de $35 \mathrm{~cm}$ ), caso o escolar náo consiga realizar o primeiro salto com essas alturas, respectivas às idades, o salto começará do nível zero. A cada acerto com cada membro inferior é adicionado mais uma espuma.

A terceira tarefa - Salto Lateral - avalia a velocidade em saltos alternados. Os escolares pulam de um lado para o outro, em uma área de $60 \times 50 \times 0,8 \mathrm{~cm}$, contornado por uma fita adesiva, com um sarrafo divisório de $60 \times 4$ x $2 \mathrm{~cm}$ durante 20 segundos e o avaliador conta quantos saltos a criança realiza.

A última tarefa - Transferência Sobre Plataforma avalia a lateralidade e a estruturação espaço-temporal. São 
utilizadas duas plataformas de madeira com medidas de $25 \times 25 \times 5 \mathrm{~cm}$, a criança se transfere de uma plataforma para a outra em 20 segundos, e conta-se quantas vezes o escolar trocou de plataforma.

A aplicação da escala de equilíbrio pediátrica - versão modificada da escala de Berg e do teste de coordenação corporal - KTK, foi realizada pelo mesmo examinador, que foi único no estudo.

\section{Análise Estatística}

Para as medidas antropométricas e idade realizou-se a análise descritiva. Empregou-se o teste Kolmegarov-Smirnov para verificar a normalidade dos dados após testar a normalidade dos dados, utilizou-se o teste $\mathrm{t}$ de Student na comparação das variáveis: escore do nível de atividade física (PAQ-C), escore de equilíbrio obtido pela escala de Berg, escore total obtido pelo teste KTK e horas de TV entre os grupos.

Para verificar a relação entre desenvolvimento motor e o escore do nível de atividade física utilizou-se o teste Chi-quadrado.

A diferença significativa foi estabelecida para $\mathrm{p}<0,05$. Utilizou-se o aplicativo Stata versão 6.0 para a análise dos dados.

\section{RESULTADOS}

Os grupos não diferiram quanto às medidas antropométricas (Tabela 1). No que se refere ao nível sócio econômico também não houve diferença entre os grupos $(\mathrm{p}=0,484)$. A maioria dos escolares de ambos os grupos pertencem à classe $\mathrm{C}$, de acordo com o Questionário Classificação Econômica Brasil (CCEB).

A comparação dos grupos em relação ao escore do nível de atividade física obtido pelo questionário PAQ-C revelou diferença significativa $(p=0,001)$, indicando que as crianças do grupo A foram classificadas como ativas e as crianças do grupo B como sedentárias (Tabela 2).

A comparação dos grupos em relação a horas de TV assistidas pelas crianças revelou diferença significativa ( $\mathrm{p}=0,00001)$, indicando que as crianças não praticante de atividades físicas e/ou lúdicas (grupo B) no contra período assistem mais TV (Tabela 2).

A comparação dos grupos no que se refere aos testes de equilíbrio revelou diferença estatisticamente signi- ficativa ( $\mathrm{p}=0,001)$, indicando que o grupo A apresenta maior pontuação nos teste de equilíbrio e portanto, melhor equilíbrio (Tabela 3). No grupo A apenas cinco escolares não apresentaram escore máximo, isto é 56 , quatro outras apresentaram escore de 55 e um escolar apresentou escore 54. No grupo B, dos 45 escolares, 28 apresentaram escore máximo de equilíbrio, oito escolares apresentaram escore de equilíbrio de 55 , outras oito apresentaram escore de 54 e um escolar apresentou escore de equilíbrio de Berg de 53.

A comparação dos grupos em relação ao desempenho nos testes de coordenação revelou diferen-

Tabela 1

Média e desvio padrão da idade e das medidas antropométricas (peso e altura) por grupo

\begin{tabular}{cccc}
\hline & IDADE & PESO & ALTURA \\
\hline Grupo A & $9,46 \pm 1,09$ & $37,22( \pm 11,09)$ & $1,40 \pm 0,10$ \\
Grupo B & $9,73 \pm 1,28$ & $41,4 \pm 10,40$ & $1,42 \pm 0,10$ \\
& $\mathrm{p}=0,739$ & $\mathrm{p}=0,581$ & $\mathrm{p}=0,128$ \\
\hline
\end{tabular}

Tabela 2

Média, desvio padrão (DP) e intervalo de confiança dos grupos A (praticantes de atividades física elou lúdica no contra periodo, ou seja extra-escolar) e B (não praticantes de atividades física elou lúdica no contra periodo, ou seja extra-escolar) em relação ao nivel de atividade física e horas assistindo TV

\begin{tabular}{cccc}
\hline Variáveis & Grupo & média \pm DP & $\begin{array}{c}\text { Intervalo de } \\
\text { confiança (95\%) }\end{array}$ \\
\hline $\begin{array}{c}\text { Nível de atividade } \\
\text { física (PAQ-C) }\end{array}$ & $\mathrm{A}$ & $3,06 \pm 0,67$ & $2,87-3,24$ \\
\hline Boras assistindo & $\mathrm{B}$ & $2,4 \pm 0,51$ & $2,25-2,56$ \\
TV & $\mathrm{B}$ & $2,59 \pm 1,32$ & $2,23-2,95$ \\
\hline
\end{tabular}

Tabela 3

Média, desvio padrão (DP) e valores mínimos (Min) e máximo (Máx) dos grupos $A$ (praticantes de atividades física elou lúdica no contra periodo, ou seja, extra-escolar) e $B$ (não praticantes de atividades física elou lúdica no contra periodo, ou seja extra-escolar) em relação ao coeficiente motor -KTK e pontuação de equilíbrio -Berg

\begin{tabular}{cccccc}
\hline & Grupo & Média \pm DP & \multicolumn{2}{c}{ Min - Máx } \\
\hline \multirow{2}{*}{ KTK } & A & $122,42 \pm 21,26$ & 79 & -148 \\
& B & $106,00 \pm 24,39$ & $63-$ & 153 \\
\hline \multirow{2}{*}{ Berg } & A & $55,87 \pm 0,40$ & 54 & -56 \\
& B & $55,40 \pm 0,74$ & $53-$ & 56 \\
\hline
\end{tabular}


ça estatisticamente significativa $(\mathrm{p}=0,0006)$, indicando que o grupo A apresenta coeficiente motor (QM) alto $(122,59 \pm 22,03)$, uma vez que o desempenho médio dos escolares nos testes está entre $119<\mathrm{QM}<140$; o grupo B apresenta coeficiente motor baixo $(106 \pm 24,39)$, pois o desempenho médio dos escolares nos testes encontra-se entre 65<QM<106 (Tabela 3).

Não houve associação entre o nível de atividade física (PAQ-C) e o teste de coordenação KTK, para o desenvolvimento motor dos escolares $(\mathrm{p}=0,086)$ (Tabela 4). Assim como não houve associação entre o nível de atividade física (PAQ-C) e a escala modificada de Berg, que avalia o equilíbrio estático e dinâmico dos escolares $(\mathrm{p}=0,309)$ (Tabela 5).

Tabela 4

Distribuição de frequência absoluta do nivel de sedentarismo em relação a coordenação motora (KTK)

\begin{tabular}{lccccc}
\hline PAK & $\mathbf{1}$ & $\mathbf{2}$ & $\mathbf{3}$ & $\mathbf{4}$ & Total \\
\hline $\begin{array}{l}\text { Coordenaçáo } \\
\text { baixa }\end{array}$ & 11 & 20 & 12 & 2 & 45 \\
$\begin{array}{l}\text { Coordenaçáo } \\
\text { normal }\end{array}$ & 1 & 10 & 1 & 2 & 18 \\
$\begin{array}{l}\text { Coordenaçáo } \\
\text { alta }\end{array}$ & 2 & 13 & 19 & 2 & 36 \\
\hline Total & 14 & 43 & 37 & 5 & 99 \\
\hline
\end{tabular}

Tabela 5

Distribuição de frequência absoluta do nivel de sedentarismo em relação ao equilibrio (Berg)

\begin{tabular}{cccccc}
\hline $\mathbf{5 3}$ & $\mathbf{1}$ & $\mathbf{2}$ & $\mathbf{3}$ & $\mathbf{4}$ & Total \\
\hline $\mathbf{5 4}$ & 1 & 5 & 3 & 0 & 9 \\
\hline $\mathbf{5 5}$ & 0 & 4 & 7 & 1 & 12 \\
\hline $\mathbf{5 6}$ & 2 & 22 & 35 & 9 & 68 \\
\hline Total & 3 & 36 & 45 & 10 & 90 \\
\hline
\end{tabular}

\section{DISCUSSÃO}

Neste estudo o grupo A, que praticou diariamente atividades físicas e/ou lúdicas no contra período apresentou maior escore do equilíbrio obtido pela escala de Berg modificada, como também alto coeficiente motor (QM) obtido pelo teste KTK, portanto apresentam comportamento ativo.

Os escolares dos dois grupos avaliadas não diferiram em relação às medidas antropométricas. Isto revela que o peso corporal, um dos fatores, que pode interferir no desenvolvimento motor, especificamente no que se refere às habilidades envolvendo equilíbrio dinâmico e coordenação, não influenciou o desenvolvimento dos escolares da faixa etária estudada.

O nível sócio econômico, outro fator, que poderia afetar o desenvolvimento motor destes escolares não foi diferente nos dois grupos estudados. A maioria dos escolares pertence ao mesmo nível socioeconômico, o que proporciona um estilo de vida parecido. Os escolares de ambos os grupos estudam em escolas da rede pública de São Caetano do Sul ou de São Paulo, as quais possuem características similares, apesar de sitiadas em municípios distintos. A escola de São Paulo localiza-se na divisa dos dois municípios. No caso de diferentes níveis socioeconômicos poderiam ocorrer distintas formas e oportunidades de estimulação, que poderia proporcionar diferenças na aquisição de habilidades motoras.

Por outro lado, este estudo revelou que apesar dos escolares estudarem em escolas com características similares, apresentaram mesmo nível socioeconômico o que permite um estilo de vida parecido, eles diferem no nível de atividade física praticada. Os escolares do grupo A foram classificados pelo questionário PAQ-C como ativos já os do grupo B foram considerados sedentários. Esta diferença se deve ao fato da prática de atividades física el ou lúdica direcionadas no contra período escolar, o que é apenas realizada por escolares do grupo A.

$\mathrm{O}$ questionário PAQ-C é um dos instrumentos adaptados à população brasileira ${ }^{18}$, e que pode ser respondido pelos pais e/ou pela própria criança, no caso da faixa etária estudada (oito a 12 anos). Isto é interessante, uma vez que os pais não ficam boa parte do dia com os filhos, não conseguindo informar com exatidão as atividades desempenhadas no dia a dia por seus filhos, o que poderia mascarar o nível de atividade física praticado pelo escolar.

Neste estudo, o fato do escolar poder responder o PAQ-C juntamente com seus pais, possibilitou determinar o real nível de atividade física desempenhado. 
Apesar de existirem vários questionários disponíveis na literatura, muitos são respondidos apenas pelos pais e/ou responsáveis, o que pode fornecer informações errôneas a respeito do dia a dia das crianças ${ }^{19,20}$.

O questionário PAQ-C possibilitou determinar o número de horas que os escolares avaliados no estudo gastam assistindo TV. Fator este que também contribui para o comportamento sedentário. O fato dos escolares do grupo A serem mais ativos do que os do grupo B, também está relacionado ao tempo gasto em horas assistindo TV, e não somente a não realização de atividades físicas e/ou lúdicas realizadas no contra período escolar. Os escolares do grupo B praticamente gastam o dobro de horas assistindo TV quando comparadas com os do grupo A. Estes dados vão ao encontro aos dados obtidos Dumith et al. ${ }^{12}$, em adolescentes da cidade de Pelotas no Rio Grande do Sul, os quais gastam em média mais de 4 horas em atividades sedentárias, como assistir TV e jogar videogame. Outros estudos reforçam que gastar mais de duas horas assistindo TV é indicativo de comportamento sedentário ${ }^{21-23}$, fator que contribui para o comportamento sedentário dos escolares do grupo B.

Este comportamento sedentário apresentado por escolares do grupo $\mathrm{B}$, os não praticantes de atividades direcionadas no contra período escolar, reflete as mudanças socioeconômicas que a sociedade sofreu decorrente da modernização e avanços tecnológicos que proporcionaram alteraçóes e mudanças nos hábitos de vida diária dos indivíduos, modificando assim o dia a dia das crianças ${ }^{24}$.

Os resultados do teste de equilíbrio obtidos pela escala de equilíbrio pediátrica revelaram que os escolares do grupo A apresentaram escore total ao realizarem os testes de equilíbrio, e seis escolares não obtiveram o escore máximo. Diferentemente dos escolares do grupo $\mathrm{B}$, em que 17 apresentaram escores entre 55 e 53 pontos. Esse achado reforça que crianças estimuladas de forma ampla e em diversos ambientes tem mais chance de praticar atividades motoras e dominá-las facilmente. Ressalta-se ainda a importância das experiências motoras na idade de oito a 12 anos, em que as crianças refinam as habilidades motoras básicas e combinam estas habilidades a fim de adquirir habilidades específicas?.

A escala de equilíbrio de equilíbrio pediátrica ${ }^{16}$ foi elaborada para população pediátrica com desordens neurológicas. Esta escala avalia o equilíbrio por meio de tarefas desempenhadas no dia a dia das crianças. Embora, esta escala de avalição tenha sido desenvolvida para crianças com desordens neurológicas, sua aplicação possibilitou detectar alteraçóes do equilíbrio em escolares sem desordem neurológica. Esperava-se que estes escolares atingissem a pontuação máxima nesta avaliação, porém a falta de refinamento do equilíbrio, devido ao comportamento sedentário de parte dos escolares do grupo B, não permitiu que estes alcançassem o escore máximo nesta avaliação, revelando a que a falta de experiência motora, não só em termos de quantidade, mas também de qualidade dos estímulos motores a que os indivíduos são expostos influencia o equilíbrio estático e dinâmico de escolares ${ }^{25}$.

Apesar da pontuação do escore apresentada por alguns escolares não estar distante da pontuação máxima esperada, ela foi significativa em relação aos dois grupos. Vale ressaltar que as tarefas avaliadas na escala de equilíbrio pediátrica compreendem atividades do dia a dia dos escolares, como o sentar e levantar, o alcance funcional. Portanto, estes resultados nos alertam para a prevenção de alteraçóes de equilíbrio, as quais na vida adulta podem levar a quedas em atividades de vida diária e/ou funcionais. Desse modo, há a necessidade de prevenção desde a infância e adolescência.

$\mathrm{O}$ teste KTK, que avaliou a coordenação motora nos escolares, fornece o QM que é um indicador global da capacidade de coordenação motora que resulta dos valores obtidos em cada teste da bateria KTK, e adaptada à população brasileira ${ }^{17}$. Os cálculos são efetuados, considerando os valores normativos para cada teste e ajustando os resultados a cada intervalo etário.

Os resultados obtidos pelo teste KTK, expresso pelo QM, revelou que as crianças do grupo A, que exploram um ambiente diferente da sua casa e da escola, conseguem ter melhor desempenho no equilíbrio e coordenação, uma vez que o QM obtido por estes escolares indica que os mesmos possuem alta coordenação, diferente dos escolares do grupo B, que apresentam menor exploração de outros ambientes e atividades físicas e/ou lúdicas direcionadas, e apresentaram média do QM classificada como baixa. Estes achados vão ao encontro aos achados de Collet ${ }^{26}$ e de Pelozzin et al. ${ }^{27}$ que obtiveram escores 
similares de coordenação normal a alta em crianças que praticavam atividades extraclasse, especificamente atividades esportivas, diferentemente das que não praticavam.

De fato, a hipótese que os escolares do grupo $\mathrm{A}$ apresentaria maior desempenho em termos de coordenação e equilíbrio foi confirmada, em virtude dos hábitos de vida, ou seja, comportamento ativo. Neste caso a prática de atividades física e/ou lúdica direcionada realizada por estes escolares, considerou a qualidade e quantidade das atividades praticadas, as quais foram adequadas às idades e ao desenvolvimento de cada idade.

Investigaçôes realizadas em crianças brasileiras ${ }^{28} \mathrm{e}$ portuguesas $^{8,25,29}$ também sugerem ligeira influência dos níveis de atividade física nos valores de coordenação motora. Além disso, investigaçóes realizadas com crianças que praticam modalidades esportivas individuais fortalecem as evidências, demonstrando que a maioria destas encontra-se nos níveis de boa e alta coordenação ${ }^{30}$.

Sabe-se ainda que o ambiente influencia a experiência motora não só em termos de quantidade de estimulação, mas também de qualidade dos estímulos motores a que os indivíduos são expostos. Crianças que vivem, por exemplo, em ambientes rurais, ambiente que possui grande espaço para exploração, conseguem ter melhor aproveitamento dos seus impulsos de aprendizagem, o que é um fator decisivo e diferencial para o desenvolvimento motor.

Estudo similar a este foi realizado com crianças de cinco a sete anos que no contra período escolar praticavam atividades físicas e/ou lúdicas, e revelou que as crianças praticantes de atividades motoras direcionadas apresentaram melhor desempenho nos testes de equilíbrio estático e dinâmico e de coordenação motora do que as crianças náo praticantes deste tipo de atividade no contra período escolar ${ }^{8}$. Portanto, ambos os estudos retratam a importância da experiência motora adquirida sobre o desenvolvimento das habilidades motoras tanto para crianças de cinco a sete anos como para crianças de oito a 12 anos.

Apesar dos resultados do teste de correlação entre nível de atividade física (PAQ-C) e o teste de coordenação KTK, assim como entre nível de atividade física e escala pediátrica de equilíbrio não serem significativos. Observa-se que há diferença no equilíbrio e coordenação motora entre os grupos A e B, que possuem comportamento ativo e sedentário, respectivamente.

Em relação à prática de atividade física e/ou lúdicas escolares ou ainda atividades esportivas extraclasse de crianças e adolescentes, espera-se que seja natural que os mais ativos apresentem repertórios motores mais ricos e variados. Além disso, são esperados melhores desempenhos em termos de coordenação e habilidades motoras, considerando-se que a qualidade e a quantidade da prática de atividades físicas estejam adequadas às idades e ao desenvolvimento de cada indivíduo. Embora não tenham sido encontradas associações significativas entre o nível de coordenação motora (KTK) e o nível de atividade física (PAQ-C), como também entre o equilíbrio (escala pediátrica de equilíbrio) e o PAQ-C dos escolares, observou-se melhores níveis de coordenação motora e equilíbrio nos escolares mais ativos (grupo $\mathrm{A}$ ).

Este estudo tem como limitação o não acompanhamento longitudinal do desenvolvimento motor das crianças de oito a 12 anos de ambos os grupos, o que permitiria verificar se a realização de atividade lúdicas e/ ou físicas resulta no refinamento das aquisiçôes motoras.

Outro fator limitante do estudo, é que não foi possível avaliar o equilíbrio e a coordenação destas crianças, antes de iniciarem as atividades propostas no contra período escolar. O que nos faz acreditar que inicialmente ambos os grupos apresentavam escores similares nos testes de equilíbrio e coordenação, é o relato dos pais sobre a melhora do desempenho das crianças após vivenciarem as experiências obtidas por estas atividades.

Sugere-se que outros estudos sejam realizados visando o acompanhamento longitudinal destas crianças, o que permitirá também uma comparação entre as idades.

\section{CONCLUSÃO}

Conclui-se que os hábitos de vida de crianças de 8 a 12 anos, principalmente no que se refere a um estilo de vida ativo, observado pela prática de atividades físicas direcionada e/ou lúdica pode influenciar o desenvolvimento de habilidades motoras em crianças desta idade. 


\section{REFERÊNCIAS}

1.Connolly K. Desenvolvimento motor: passado presente e futuro. Rev Paul Educ Fís 2000;3:6-15.

2.Passaglio TK, Barros Neto R, Andrade MC. Investigaçáo teórico-prática do desenvolvimento motor de crianças de 2 a 11 anos. Rev Neurocienc 2006;3(1):23-33.

3.Bessa SFM, Pereira SJ. Equilíbrio e coordenação motora em pré-escolares: um estudo comparativo. Rev Bras Ciên e Mov 2002;10(4):57-62.

4.Santos S, Dantas L, Oliveira JA. Desenvolvimento motor de crianças, de idosos, e de pessoas com transtorno da coordenação. Rev Paul Educ Fis 2004;18:33-44.

5.Rossi ACS, Caetano MJD, Siveira CRA, Marques I, Gobbi TB. Atividade física extra-escolar e desenvolvimento motor de crianças. Temas sobre desenvolvimento motor 2007; 15:75-81.

6.Rosa Neto F, Santos APM, Xavier RFC, Amaro KN. A Importância da avaliação motora em escolares: análise da confiabilidade da Escalade Desenvolvimento Motor. Rev Bras Cineantropom Desempenho Hum 2010;12(6):422427.

\section{http://dx.doi.org/10.5007/1980-0037.2010v12n6p422}

7.Carvalho B, Sá CSC, Deliberato PCP. Influência da prática lúdica no equilíbrio e na coordenação motora de crianças. Rev Bras Cienc Saúde 2008;18:3-12. 8.Faustino AJD, Proença MJ, Silva RJDM, Almeida SPPD. Aptidão motora e coordenaçáo corporal em alunos do $3^{\circ}$ ano do $1^{\circ}$ ciclo do ensino básico. Rev Dep Educ Fís e Artística 2004;5:97-104.

9.Stabelini NA, Mascarenhas LPG, Nunes, FG, Lepre C, Campos C. Relação entre fatores ambientais e habilidades motoras bascas em crianças de 6 e 7 anos. Rev. Mackenzie de Educ. Fís e Esport 2004;3:135-140.

10.Berleze A, Haeffner LSB, Valentini NC. Desempenho motor de crianças obesas: uma investigação do processo e produto de habilidades motoras fundamentais. Rev. Bras Cineantropom Desempenho Hum 2007;9(2):134-144.

11. Catenassi FZ, Marques I, Bastos CB, Basso L, Ronque VER, Gerage AM. Relação entre índice de massa corporal e habilidade motora grossa em crianças de quatro a seis anos. Rev Bras Med Esport 2007;13(4):227-230.

http://dx.doi.org/10.1590/S1517-86922007000400003

12.Dumith SC, Hallal PC, Menezes AMB, Araújo CL. Sedentary behavior in adolescents: the 11-year follow-up of the 1993 Pelotas (Brazil) birth cohort study. Cad. Saúde Pública 2010; 26(10):1928-1936.

http://dx.doi.org/10.1590/S0102-311X2010001000009

13.Andersen LB, Bugge A, Dencker M, Eiberg S, El-Naaman B. The association between physical activity, physical fitness and development of metabolic disorders. Int J Pediatr Obes 2011;6 Suppl 1:29-34.

http://dx.doi.org/10.3109/17477166.2011.606816

14.Lambourne K, Donnelly JE. The role of physical activity in pediatric obesity. Pediatr Clin North Am 2011;58(6):1481-91.

http://dx.doi.org/10.1016/j.pcl.2011.09.004

15.Franjoine MR, Gunther JS, Taylor MJ. Pediatric balance scale: a modified version of the Berg balance scale for the school-age child with mild to moderate motor impairment. Pediatr Phys Pher 2003;15:114-128.

http://dx.doi.org/10.1097/01.PEP.0000068117.48023.18

16.Ribeiro AS, David AC, Barbacena MM, Rodrigues ML, França NM. Teste de Coordenação Corporal para Crianças (KTK): aplicaçôes e estudos normati- vos. Motri. 2012,8(3),40-51

17.Gorla JI, Araujo PF, Carminato RA. Desempenho psicomotor em portadores de deficiência mental: avaliação e intervenção. Rev Bras Cienc Esport 2004;25(3):133-147.

18.Silva RCR, Malina RM. Nível de atividade física em adolescentes do município de Niterói, Rio de Janeiro, Brasil. Cad Saúde Pública 2000;16(4):10911997.

http://dx.doi.org/10.1590/S0102-311X2000000400027

19.Hallal PC, Dumith SC, Bastos JP, Reichert FF, Siqueira FV, Azevedo MR. Evolução da pesquisa epidemiológica em atividade física no Brasil: revisão sistemática. Rev Saúde Pública 2007;41:453-60.

http://dx.doi.org/10.1590/S0034-89102007000300018

20.Reichert FF, Menezes AMB, Araújo CL, Hallal PC. Self-reporting versus parental reporting ofphysical activity in adolescents: the 11-yearfollow-up of the 1993 Pelotas (Brazil) birthcohort study. Cad. Saúde Pública 2012;26(10):1921-1927.

http://dx.doi.org/10.1590/S0102-311X2010001000008

21.Brodersen NH, Steptoe A, Williamson S, Wardle J. Sociodemographic, developmental, environmental, and psychological correlates of physical activity and sedentary behavior at age 11 to 12. Ann Behav Med 2005;29:2-11.

http://dx.doi.org/10.1207/s15324796abm2901_2

22.Hesketh K, Wake M, Graham M, Waters E. Stability of television viewing and electronic game/computer use in a prospective cohort study of Australian children: relationship with body mass index. Int J Behav Nutr Phys Act 2007;4:60.

http://dx.doi.org/10.1186/1479-5868-4-60

23.Araújo CL, Menezes AMB, Vieira MFA, Neutzling MB, Gonçalves H, Anselmi L, et al. The 11-year follow-up of the 1993 Pelotas (Brazil) birth cohort study: methods. Cad Saúde Pública 2010;26:1875-86.

http://dx.doi.org/10.1590/S0102-311X2010001000003

24.Luz GM, Raymundo LS, Kuhnen A. Uso dos espaços urbanos pelas crianças: umarevisão. Rev Psicologia: Teoria e Prática 2010;12(3):172-184.

25.Lopes VP, Maia JAR. Efeitos do ensino no desenvolvimento da capacidade de coordenação corporal em crianças de 8 anos de idade. Rev Paul Educ Fís 1997;11(1):40-48.

26.Collet C, Folle A, Pellozin, Botti M, Nascimento JV. Nível de coordenação motora de escolares da rede estadual da cidade de Florianópolis. Motriz 2008;14(4):373-380.

27.Pelozin F, Folle A, Collet C, Botti M, Nascimento JV. Nível de coordenação motora de escolares de 09 a 11 anos da rede estadual de ensino da cidade de Florianópolis/SC. Rev Mackenzie Educ Fís e Esport 2009;8(2):123-132.

28.Elias RGM, Silva PG, Fernandes CAM, Rinaldi W. Estudo comparativo da coordenação entre crianças praticantes e não praticantes de atividade física além do ensino regular. Rev da Educ Fís/UEM 2007;18:24-27.

29.Lopes VP, Maia JAR, Silva RG, Morais FP. Estudo do nível de desenvolvimento da coordenação motora da população escolar ( 6 a 10 anos de idade) da Regiāo Autônoma dos Açores. Rev Port Cien Desp 2003;3(1):47-60.

30.Deus RKBC, Bustamante A, Lopes VP, Seabra A, Silva RG, Maia JAR. Coordenaçấo motora: um estudo de tracking em crianças dos 6 aos 10 anos da Região Autónoma dos Açores, Portugal. Rev Bras Cineantropom Desempenho Hum 2008;10(3):215-222. 